\title{
THE CAFFARELLI-KOHN-NIRENBERG INEQUALITIES ON COMPLETE MANIFOLDS
}

\author{
Changyu Xia
}

\begin{abstract}
We find a new sharp Caffarelli-Kohn-Nirenberg inequality and show that the Euclidean spaces are the only complete non-compact Riemannian manifolds of nonnegative Ricci curvature satisfying this inequality. We also show that a complete open manifold with non-negative Ricci curvature in which the optimal Nash inequality holds is isometric to a Euclidean space.
\end{abstract}

\section{Introduction}

Let $C_{0}^{\infty}\left(\mathbb{R}^{n}\right)$ be the space of smooth functions with compact support in the $n$ dimensional Euclidean space $\mathbb{R}^{n}$. Among a much more general family of inequalities, Caffarelli, Kohn and Nirenberg proved the following result.

Theorem 1.1. ([CKN]) Let $n \geq 2, r>p>1$, and $\alpha, \beta$ be fixed real numbers satisfying

$$
\frac{1}{p}+\frac{\alpha}{n}, \frac{p-1}{p(r-1)}+\frac{\beta}{n}, \frac{1}{r}+\frac{\gamma}{n}>0
$$

where

$$
\gamma=\frac{1}{r}(\alpha-1)+\frac{p-1}{p r} \beta
$$

There exists a positive constant $C$ such that the following inequality holds for all $f \in C_{0}^{\infty}\left(\mathbb{R}^{n}\right)$

$$
\begin{aligned}
& \int_{\mathbb{R}^{n}}|x|^{\gamma r}|f|^{r} d x \\
\leq & C\left(\int_{\mathbb{R}^{n}}|x|^{\alpha p}|\nabla f|^{p} d x\right)^{\frac{1}{p}}\left(\int_{\mathbb{R}^{n}}|x|^{\beta}|f|^{\frac{p(r-1)}{p-1}} d x\right)^{\frac{p-1}{p}},
\end{aligned}
$$

where $|x|$ is the Euclidean length of $x \in R^{n}$.

In this paper, we obtain the exact value of the smallest admissible constant $C$ in (1.3) for some special cases. Namely, we have

Received by the editors June 15, 2006.

2000 Mathematics Subject Classification. 53C20, 53C21, 57R70, 31C12.

Key words and phrases. Sharp Caffarelli-Kohn-Nirenberg inequalities, complete manifolds, nonnegative Ricci curvature, Euclidean space, Nash inequality.

This work was partially supported by CNPq and PRONEX. 
Theorem 1.2. Let $n, r, p, \alpha, \beta, \gamma$ be as in Theorem 1.1. Then for all $f \in$ $C_{0}^{\infty}\left(\mathbb{R}^{n}\right)$, we have

$$
\begin{aligned}
& \int_{\mathbb{R}^{n}}|x|^{\gamma r}|f|^{r} d x \\
\leq & \frac{r}{n+\gamma r}\left(\int_{\mathbb{R}^{n}}|x|^{\alpha p}|\nabla f|^{p} d x\right)^{\frac{1}{p}}\left(\int_{\mathbb{R}^{n}}|x|^{\beta}|f|^{\frac{p(r-1)}{p-1}} d x\right)^{\frac{p-1}{p}} .
\end{aligned}
$$

Moreover, when

$$
n+\beta<\left(1-\alpha+\frac{\beta}{p}\right) \frac{(r-1) p}{r-p},
$$

the inequality (1.4) is best possible in the sense that

$$
\begin{aligned}
\operatorname{linf}_{f \in C_{0}^{\infty}\left(\mathbb{R}^{n}\right)-\{0\}} & \frac{\left(\int_{\mathbb{R}^{n}}|x|^{\alpha p}|\nabla f|^{p} d x\right)^{\frac{1}{p}}\left(\int_{\mathbb{R}^{n}}|x|^{\beta}|f|^{\frac{p(r-1)}{p-1}} d x\right)^{\frac{p-1}{p}}}{\int_{\mathbb{R}^{n}}|x|^{\gamma r}|f|^{r} d x} \\
= & \frac{n+\gamma r}{r}
\end{aligned}
$$

and a family of minimizers of (1.6) is given by

$$
g(x)=\left(\lambda+|x|^{1-\alpha+\frac{\beta}{p}}\right)^{\frac{1-p}{r-p}}, \lambda>0 .
$$

In the next part of the present paper, we study complete manifolds with nonnegative Ricci curvature in which the Caffarelli-Kohn-Nirenberg type inequality (1.4) is satisfied. For a Riemannian manifold $M$, we let $d v$ be the Riemannian volume element on $M$, denote by $\nabla$ the gradient operator, $C_{0}^{\infty}(M)$ the space of smooth functions on $M$ with compact support, $B(x, r)$ the geodesic ball with center $x \in M$ and radius $r$ and $\operatorname{vol}[B(p, r)]$ be the volume of $B(p, r)$.

Theorem 1.3. Let $n, r, p, \alpha, \beta, \gamma$ be as in Theorem 1.1 and assume that (1.5) holds. Let $M$ be an $n$-dimensional complete open Riemannian manifold with nonnegative Ricci curvature. Fix a point $x_{0} \in M$ and denote by $\mu$ the distance function on $M$ from $x_{0}$. If for any $f \in C_{0}^{\infty}(M)$, we have

$$
\begin{aligned}
& \int_{M} \mu^{\gamma r}|f|^{r} d v \\
\leq & \frac{r}{n+\gamma r}\left(\int_{M} \mu^{\alpha p}|\nabla f|^{p} d v\right)^{\frac{1}{p}}\left(\int_{M} \mu^{\beta}|f|^{\frac{p(r-1)}{p-1}} d v\right)^{\frac{p-1}{p}} .
\end{aligned}
$$

Then $M$ is isometric to $\mathbb{R}^{n}$.

The sharp logarithmic Sobolev inequalities or entropy-energy inequalities in Euclidean space states that for any $f \in C_{0}^{\infty}\left(\mathbb{R}^{n}\right)$ with $\int_{\mathbb{R}^{n}} f^{2} d x=1$ it holds (cf. [D], [B])

$$
\int_{\mathbb{R}^{n}} f^{2} \log f^{2} d x \leq \frac{n}{2} \log \left(\frac{2}{n \pi e} \int_{\mathbb{R}^{n}}|\nabla f|^{2} d x\right) .
$$


It has been shown by Bakry, Concordet and Ledoux that (cf. [BCL]) a complete $n$-dimensional Riemannian manifold $M$ with non-negative Ricci curvature satisfying the optimal logarithmic Sobolev inequality, i.e.

$$
\begin{aligned}
& \int_{M} f^{2} \log f^{2} d v \leq \frac{n}{2} \log \left(\frac{2}{n \pi e} \int_{M}|\nabla f|^{2} d v\right), \\
& \forall f \in C_{0}^{\infty}(M) \text { with } \int_{M} f^{2} d v=1,
\end{aligned}
$$

is isometric to $\mathbb{R}^{n}$.

A similar result for the Sobolev inequality was obtained by Ledoux in [Le] and he showed that a complete $n$-dimensional Riemannian manifold $M$ with nonnegative Ricci curvature in which one of the Sobolev inequalities

$$
\begin{aligned}
& \left(\int_{M}|f|^{p} d v\right)^{1 / p} \leq C\left(\int_{M}|\nabla f|^{q} d v\right)^{1 / q}, \\
& \forall f \in C_{0}^{\infty}(M), 1 \leq q<n, \frac{1}{p}=\frac{1}{q}-\frac{1}{n},
\end{aligned}
$$

is satisfied with $C$ the optimal constant of this inequality in $\mathbb{R}^{n}$ is isometric to $\mathbb{R}^{n}$. This theorem of Ledoux has been generalized in [X1].

Another important inequality is the so called Nash inequality stating that for any $f \in C_{0}^{\infty}\left(\mathbb{R}^{n}\right)$ we have

$$
\left(\int_{\mathbb{R}^{n}} f^{2} d v\right)^{1+2 / n} \leq C_{n}\left(\int_{\mathbb{R}^{n}}|\nabla f|^{2} d v\right)\left(\int_{\mathbb{R}^{n}}|f| d v\right)^{4 / n}
$$

for a constant $C_{n}$ depending only on $n$. This inequality is a particular case of the Gagliardo-Nirenberg inequalities for which numerous applications have been found. It has been shown by Carlen and Loss [CL] that the best possible constant in (1.7) is given by

$$
C_{n}=\frac{2((n+2) / 2)^{(n+2) / n}}{n \lambda_{1}^{N}\left(B_{n}\right)\left|B_{n}\right|^{2 / n}}
$$

where $\left|B_{n}\right|$ denotes the volume of the unit ball in $\mathbb{R}^{n}$, and where $\lambda_{1}^{n}\left(B_{n}\right)$ denotes the first nonzero Neumann eigenvalue of the Laplacian operator on $B_{n}$.

In [L1], Ledoux proposed the problem that if a complete $n$-manifold of non-negative Ricci curvature in which the Nash inequality (1.7) holds with $C_{n}$ given by (1.8) is isometric to $\mathbb{R}^{n}$. With respect to this problem, Druet, Hebey and Vaugon showed in [DHV] that a complete $n$-dimensional manifold $M$ with non-negative Ricci curvature in which the following Nash inequality holds:

$$
\left(\int_{M} f^{2} d v\right)^{1+\frac{2}{n}} \leq C_{n}\left(\int_{M}|f| d v\right)^{\frac{4}{n}} \int_{M}|\nabla f|^{2} d v, \quad \forall f \in C_{0}^{\infty}(M),
$$

is flat, where $C_{n}$ is given by (1.9).

In this paper, using the result proved by Druet, Hebey and Vaugon [DVH], we show that the problem by Ledoux has a positive answer. 
Theorem 1.4. Let $M$ be an complete $n$-dimensional manifold $M$ with non-negative Ricci curvature. If for any $f \in C_{0}^{\infty}(M)$, it holds

$$
\left(\int_{M} f^{2} d v\right)^{1+\frac{2}{n}} \leq C_{n}\left(\int_{M}|f| d v\right)^{\frac{4}{n}} \int_{M}|\nabla f|^{2} d v
$$

where $C_{n}$ is given by (1.9), then $M$ is isometric to $\mathbb{R}^{n}$.

Complete manifolds with non-negative Ricci curvature in which some other type Caffarelli-Kohn-Nirenberg ineqaulity (cf. [CKN], [CCh], [L], [CW]) holds were studied in [CX1]. The structure of complete manifolds with non-negative Ricci curvature in which some Gagliardo-Nirenberg type ineqaulity (cf. [DPD1], [DPD2]) holds has been studied recently in [X3]. For some interesting results about complete manifolds with non-negative Ricci curvature, we refer to $[\mathrm{AG}],[\mathrm{A}],[\mathrm{CX} 1],[\mathrm{CC}],[\mathrm{Li}],[\mathrm{SS}],[\mathrm{S} 1],[\mathrm{S} 2]$, [SSO], [SO1], [SO2], [W], [X2].

\section{A Proof of Theorem 1.2}

Let $\Delta$ be the Laplacian operator on $\mathbb{R}^{n}$; then for the position vector $x$ in $\mathbb{R}^{n}$, it holds $\Delta|x|^{2}=2 n$. For any $f \in C_{0}^{\infty}\left(\mathbb{R}^{n}\right)$, since $|\nabla| x||=1$ almost everywhere, it follows from the divergence theorem that

$$
\begin{aligned}
& \int_{\mathbb{R}^{n}}|x|^{\gamma r}|f|^{r} d x \\
= & \frac{1}{2 n} \int_{\mathbb{R}^{n}}|f|^{r}|x|^{\gamma r} \Delta|x|^{2} d x \\
= & -\frac{1}{2 n} \int_{\mathbb{R}^{n}}\left\langle\nabla\left(|x|^{2}\right), \nabla\left(|f|^{r}|x|^{\gamma r}\right)\right\rangle d x \\
= & -\left.\frac{\gamma r}{n} \int_{\mathbb{R}^{n}}|x|^{\gamma r}|f|^{r}|\nabla| x\right|^{2} d x+\frac{r}{n} \int_{\mathbb{R}^{n}}|x|^{\gamma r+1}\langle\nabla|x|, \nabla|f|\rangle|f|^{r-1} d x \\
= & -\frac{\gamma r}{n} \int_{\mathbb{R}^{n}}|x|^{\gamma r}|f|^{r} d x+\frac{r}{n} \int_{\mathbb{R}^{n}}|x|^{\gamma r+1}\langle\nabla|x|, \nabla|f|\rangle|f|^{r-1} d x
\end{aligned}
$$

The Schwarz inequality implies that $\langle\nabla|x|, \nabla|f|\rangle \leq|\nabla| x|| \cdot|\nabla| f||=|\nabla| f||$ almost everywhere. Since $|\nabla| f||=|\nabla f|$ almost everywhere, we conclude from (2.1) that

$$
\int_{\mathbb{R}^{n}}|x|^{\gamma r}|f|^{r} d x \leq-\frac{\gamma r}{n} \int_{\mathbb{R}^{n}}|x|^{\gamma r}|f|^{r} d x+\frac{r}{n} \int_{\mathbb{R}^{n}}|x|^{\gamma r+1}|\nabla f||f|^{r-1} d x,
$$

which implies that

$$
\begin{aligned}
& \int_{\mathbb{R}^{n}}|x|^{\gamma r}|f|^{r} d x \\
\leq & \frac{r}{n+\gamma r} \int_{\mathbb{R}^{n}}|x|^{\gamma r+1}|\nabla f||f|^{r-1} d x \\
= & \frac{r}{n+\gamma r} \int_{\mathbb{R}^{n}}|x|^{\alpha}|\nabla f||x|^{\frac{(p-1) \beta}{p}}|f|^{r-1} d x \\
\leq & \frac{r}{n+\gamma r}\left(\int_{\mathbb{R}^{n}}|x|^{\alpha p}|\nabla f|^{p} d x\right)^{\frac{1}{p}}\left(\int_{\mathbb{R}^{n}}|x|^{\beta}|f|^{\frac{p(r-1)}{p-1}} d x\right)^{\frac{p-1}{p}},
\end{aligned}
$$

where in the last inequality, we used the Hölder's inequality. Thus (1.4) holds. 
To conclude the proof of Theorem 1.2, it suffices to check that when

$$
g(x)=\left(\lambda+|x|^{1-\alpha+\frac{\beta}{p}}\right)^{\frac{1-p}{r-p}}, \quad \lambda>0,
$$

then

$$
\frac{\left(\int_{\mathbb{R}^{n}}|x|^{\alpha p}|\nabla g|^{p} d x\right)^{\frac{1}{p}}\left(\int_{\mathbb{R}^{n}}|x|^{\beta}|g|^{\frac{p(r-1)}{p-1}} d x\right)^{\frac{p-1}{p}}}{\int_{\mathbb{R}^{n}}|x|^{\gamma r}|g|^{r} d x}=\frac{n+\gamma r}{r} .
$$

For $\lambda \in(0,+\infty)$, set

$$
\begin{aligned}
& A(\lambda)=\frac{(p-1)\left(1-\alpha+\frac{\beta}{p}\right)}{r-p} \int_{\mathbb{R}^{n}}|x|^{\beta}\left(\lambda+|x|^{1-\alpha+\frac{\beta}{p}}\right)^{\frac{(1-r) p}{r-p}} d x, \\
& B(\lambda)=\int_{\mathbb{R}^{n}}|x|^{\alpha-1+\left(1-\frac{1}{p}\right) \beta}\left(\lambda+|x|^{1-\alpha+\frac{\beta}{p}}\right)^{\frac{(1-p) r}{r-p}} d x .
\end{aligned}
$$

We claim that the functions $A$ and $B$ are well defined. In fact, we have

$$
\begin{aligned}
A(1) & =\frac{(p-1)\left(1-\alpha+\frac{\beta}{p}\right)}{r-p} \int_{\mathbb{R}^{n}}|x|^{\beta}\left(1+|x|^{1-\alpha+\frac{\beta}{p}}\right)^{\frac{(1-r) p}{r-p}} d x \\
& =\frac{(p-1)\left(1-\alpha+\frac{\beta}{p}\right)}{r-p} \int_{0}^{+\infty} c_{n-1} r^{\beta+n-1}\left(1+r^{1-\alpha+\frac{\beta}{p}}\right)^{\frac{(1-r) p}{r-p}} d r
\end{aligned}
$$

where $c_{n-1}$ denotes the area of the unit sphere in $R^{n}$. Since

$$
\frac{p-1}{p(r-1)}+\frac{\beta}{n}>0
$$

one has

$$
\beta+n-1>-1 .
$$

It follows from (1.5) and (2.4) that $A(1)$ converges. On the other hand, one has

$$
\begin{aligned}
B(1) & =\int_{\mathbb{R}^{n}}|x|^{\alpha-1+\left(1-\frac{1}{p}\right) \beta}\left(1+|x|^{1-\alpha+\frac{\beta}{p}}\right)^{\frac{(1-p) r}{r-p}} d x \\
& =\int_{0}^{\infty} c_{n-1} r^{n-1+\alpha-1+\left(1-\frac{1}{p}\right) \beta}\left(1+r^{1-\alpha+\frac{\beta}{p}}\right)^{\frac{(1-p) r}{r-p}} d r .
\end{aligned}
$$

Since $n+\gamma r>0$, we have

$$
n-1+\alpha-1+\left(1-\frac{1}{p}\right) \beta>-1 .
$$

Also, it is easy to see that (1.5) is equivalent to

$$
n-1+\alpha-1+\left(1-\frac{1}{p}\right) \beta+\left(1-\alpha+\frac{\beta}{p}\right) \frac{(1-p) r}{r-p}<-1 .
$$

Thus $B(1)$ converges. It is easy to see that

$$
A(\lambda)=\lambda^{\frac{n+\gamma r}{1-\alpha+\beta / p}-\frac{r(p-1)}{r-p}} \cdot A(1), \quad B(\lambda)=\lambda^{\frac{n+\gamma r}{1-\alpha+\beta / p}-\frac{r(p-1)}{r-p}} \cdot B(1) .
$$

So our claim is true. 
Since

$$
\nabla g=\frac{(1-p)\left(1-\alpha+\frac{\beta}{p}\right)}{r-p}|x|^{-\alpha+\frac{\beta}{p}}\left(\lambda+|x|^{1-\alpha+\frac{\beta}{p}}\right)^{\frac{1-r}{r-p}} \nabla|x|,
$$

one can easily obtain that

$$
\left(\int_{\mathbb{R}^{n}}|x|^{\alpha p}|\nabla g|^{p} d x\right)^{\frac{1}{p}}\left(\int_{\mathbb{R}^{n}}|x|^{\beta}|g|^{\frac{p(r-1)}{p-1}} d x\right)^{\frac{p-1}{p}}=A(\lambda),
$$

and that

$$
\int_{\mathbb{R}^{n}}|x|^{\gamma r}|g|^{r} d x=B(\lambda)
$$

In order to see that (2.3) holds, we need only to show that

$$
A(1)=\frac{n+\gamma r}{r} B(1) \text {. }
$$

This can be seen as follows.

$$
\begin{aligned}
& A(1) \\
= & \frac{(p-1)\left(1-\alpha+\frac{\beta}{p}\right)}{r-p} \int_{\mathbb{R}^{n}}|x|^{\beta}\left(1+|x|^{1-\alpha+\frac{\beta}{p}}\right)^{\frac{(1-r) p}{r-p}} d x \\
= & \frac{(p-1)\left(1-\alpha+\frac{\beta}{p}\right)}{r-p}\left(\int_{\mathbb{R}^{n}}|x|^{\alpha-1+\left(1-\frac{1}{p}\right) \beta}\left(1+|x|^{1-\alpha+\frac{\beta}{p}}\right)^{\frac{r(1-p)}{r-p}} d x\right. \\
& \left.-\int_{\mathbb{R}^{n}}|x|^{\alpha-1+\left(1-\frac{1}{p}\right) \beta}\left(1+|x|^{1-\alpha+\frac{\beta}{p}}\right)^{\frac{p(1-r)}{r-p}} d x\right) \\
= & \frac{(p-1)\left(1-\alpha+\frac{\beta}{p}\right)}{r-p}\left(B(1)+\frac{r-p}{r(p-1)} B^{\prime}(1)\right) .
\end{aligned}
$$

By (2.6),

$$
B^{\prime}(1)=\left(\frac{n+\gamma r}{1-\alpha+\beta / p}-\frac{r(p-1)}{r-p}\right) B(1)
$$

Combining (2.10) and (2.11), one gets (2.9). This completes the proof of Theorem 1.2 .

\section{Proofs of Theorems 1.3 and 1.4}

Proof of Theorem 1.3. Let $\theta>1$ be fixed and set $\rho=\theta^{-1} \mu$. For any $\lambda>0$, let

$$
F(\lambda)=\int_{M} \frac{\rho^{\alpha-1+\left(1-\frac{1}{p}\right) \beta} d v}{\left(\lambda+\rho^{1-\alpha+\frac{\beta}{p}}\right)^{\frac{r(p-1)}{r-p}}} .
$$

Let us first verify that $F$ is a well defined $C^{1}$ function on $(0,+\infty)$. In fact, by Fubini theorem (cf. [SY])

$$
F(\lambda)=\int_{0}^{+\infty} \operatorname{vol}\left\{x: \frac{\rho^{\alpha-1+\left(1-\frac{1}{p}\right) \beta}}{\left(\lambda+\rho^{1-\alpha+\frac{\beta}{p}}\right)^{\frac{r(p-p)}{r-p}}}(x)>s\right\} d s .
$$


Since $M$ has non-negative Ricci curvature, the Bishop-Gromov comparison theorem implies that $\operatorname{vol}\left[B\left(x_{0}, t\right)\right] \leq\left|B_{n}\right| t^{n}$ (cf. $[\mathrm{BC}],[\mathrm{G}]$ ). Set

$$
\omega=1-\alpha+\left(\frac{1}{p}-1\right) \beta, z=\frac{r(p-1)}{r-p},
$$

then we have from (1.5) that

$$
n-\omega-1-z(\omega+\beta)<-1 .
$$

Making the variable change

$$
s=\frac{t^{\alpha-1+\left(1-\frac{1}{p}\right) \beta}}{\left(\lambda+t^{1-\alpha+\frac{\beta}{p}}\right)^{\frac{r(p-1)}{r-p}}}
$$

in (3.1), we get

$$
\begin{aligned}
F(\lambda) & =\int_{0}^{+\infty} \operatorname{vol}\left[B\left(x_{0}, \theta t\right)\right] \cdot \frac{\omega \lambda+(\omega+z(\omega+\beta)) t^{\omega+\beta}}{t^{\omega+1}\left(\lambda+t^{\omega+\beta}\right)^{z+1}} d t \\
& \leq \int_{0}^{+\infty} \frac{\left|B_{n}\right| \theta^{n}\left(\omega \lambda+(\omega+z(\omega+\beta)) t^{\omega+\beta}\right) t^{n-\omega-1}}{\left(\lambda+t^{\omega+\beta}\right)^{z+1}} d t .
\end{aligned}
$$

Since $n-\omega-1=n+\gamma r-1>-1$ and (3.2) holds, we know that $0 \leq F(\lambda)<$ $+\infty, \forall \lambda>0$, that $F$ is differentiable and that

$$
F^{\prime}(\lambda)=-\frac{r(p-1)}{r-p} \int_{M} \frac{\rho^{\alpha-1+\left(1-\frac{1}{p}\right) \beta} d v}{\left(\lambda+\rho^{1-\alpha+\frac{\beta}{p}}\right)^{\frac{p(r-1)}{r-p}}}
$$

Observe that $|\nabla \rho|=\theta^{-1}$ almost everywhere. By an approximation procedure, we can apply (1.7) to $\left(\lambda+\rho^{1-\alpha+\beta / p}\right)^{-\frac{p-1}{r-p}}$ for every $\lambda>0$ to get

$$
\begin{aligned}
& F(\lambda) \\
= & \int_{M} \frac{\rho^{\alpha-1+\left(1-\frac{1}{p}\right) \beta} d v}{\left(\lambda+\rho^{\left.1-\alpha+\frac{\beta}{p}\right)^{\frac{r(p-1)}{r-p}}}\right.} \\
\leq & \frac{r(p-1)(1-\alpha+\beta / p)}{(n+\gamma r)(r-p)} \int_{M} \frac{\rho^{\beta} d v}{\left(\lambda+\rho^{1-\alpha+\frac{\beta}{p}}\right)^{\frac{p(r-1)}{r-p}}} \\
= & \frac{r(p-1)(1-\alpha+\beta / p)}{(n+\gamma r)(r-p)} \int_{M} \frac{\rho^{\alpha-1+\left(1-\frac{1}{p}\right) \beta}\left(\rho^{1-\alpha+\frac{\beta}{p}}+\lambda-\lambda\right) d v}{\left(\lambda+\rho^{1-\alpha+\frac{\beta}{p}}\right)^{\frac{p(r-1)}{r-p}}} \\
= & \frac{r(p-1)(1-\alpha+\beta / p)}{(n+\gamma r)(r-p)}\left(F(\lambda)+\frac{r-p}{r(p-1)} \lambda F^{\prime}(\lambda)\right)
\end{aligned}
$$

Thus we have

$$
-\frac{1-\alpha+\beta / p}{n+\gamma r} \lambda F^{\prime}(\lambda) \leq\left(\frac{r(p-1)(1-\alpha+\beta / p)}{(n+\gamma r)(r-p)}-1\right) F(\lambda),
$$


or

$$
-\lambda F^{\prime}(\lambda) \leq l F(\lambda)
$$

where

$$
\begin{aligned}
l & =\frac{r(p-1)}{r-p}-\frac{n+\gamma r}{(1-\alpha+\beta / p)} \\
& =\frac{r(p-1)}{r-p}-\frac{n+\beta}{(1-\alpha+\beta / p)}-1 \\
& =\frac{p(r-1)(1-\alpha+\beta / p)-(r-p)(n+\beta)}{(1-\alpha+\beta / p)(r-p)} \\
& >0,
\end{aligned}
$$

where in the last inequality, we used (1.5).

Consider the function $B:(0,+\infty) \rightarrow \mathbb{R}$ introduced in the last section. By $(2.6)$

$$
B(\lambda)=\lambda^{-l} \cdot B(1)
$$

and so

$$
-\lambda B^{\prime}(\lambda)=l B(\lambda)
$$

We claim that if for some $\lambda_{0}>0, F\left(\lambda_{0}\right)<B\left(\lambda_{0}\right)$, then $F(\lambda)<B(\lambda), \forall \lambda \in\left(0, \lambda_{0}\right]$. In order to see this, suppose that there exists some $\tilde{\lambda} \in\left(0, \lambda_{0}\right)$ such that $F(\tilde{\lambda}) \geq B(\tilde{\lambda})$. Set

$$
\lambda_{1}=\sup \left\{\lambda<\lambda_{0} ; F(\lambda)=B(\lambda)\right\} .
$$

Then for any $\lambda \in\left[\lambda_{1}, \lambda_{0}\right], 0<F(\lambda) \leq B(\lambda)$ and so we have from (3.7) and (3.9) that

$$
\lambda\left(F^{\prime}(\lambda)-B^{\prime}(\lambda)\right) \geq l(B(\lambda)-F(\lambda)) \geq 0, \quad \forall \lambda \in\left[\lambda_{1}, \lambda_{0}\right] .
$$

It follows that the function $F(\lambda)-B(\lambda)$ is increasing on $\left[\lambda_{1}, \lambda_{0}\right]$. Consequently, we have

$$
0=(F-B)\left(\lambda_{1}\right) \leq(F-B)\left(\lambda_{0}\right)<0 .
$$

This is a contradiction. Thus the above claim is true.

Before we can finish the proof of Theorem 1.3, we will need the following lemma.

Lemma 3.1. We have

$$
\liminf _{\lambda \rightarrow 0} \frac{F(\lambda)}{B(\lambda)} \geq \theta^{n}>1 .
$$

Proof. Fix a small $\epsilon>0$. Since

$$
\lim _{u \rightarrow 0} \frac{\operatorname{vol}\left[B\left(x_{0}, u\right)\right]}{V_{0}(u)}=1,
$$

there exists a $\delta>0$ such that

$$
\operatorname{vol}\left[B\left(x_{0}, h\right)\right] \geq(1-\epsilon) V_{0}(h), \forall h \leq \frac{\delta}{\theta},
$$


where $V_{0}(h)$ denotes the volume of an $h$-ball in $\mathbb{R}^{n}$. It then follows from (3.3) that

$$
\begin{aligned}
F(\lambda) & \geq \int_{0}^{\delta / \theta} \operatorname{vol}\left[B\left(x_{0}, \theta t\right)\right] \frac{\omega \lambda+(\omega+z(\omega+\beta)) t^{\omega+\beta}}{t^{\omega+1}\left(\lambda+t^{\omega+\beta}\right)^{z+1}} d t \\
& \geq(1-\epsilon) \theta^{n} \int_{0}^{\delta / \theta} V_{0}(t) \frac{\omega \lambda+(\omega+z(\omega+\beta)) t^{\omega+\beta}}{t^{\omega+1}\left(\lambda+t^{\omega+\beta}\right)^{z+1}} d t \\
& =(1-\epsilon) \theta^{n} \lambda^{-l} \int_{0}^{\delta /\left(\theta \lambda^{\frac{1}{\omega+\beta}}\right)} V_{0}(s) \frac{\omega+(\omega+z(\omega+\beta)) s^{\omega+\beta}}{s^{\omega+1}\left(1+s^{\omega+\beta}\right)^{z+1}} d s .
\end{aligned}
$$

On the other hand, it is easy to see that

$$
B(\lambda)=\lambda^{-l} \int_{0}^{+\infty} V_{0}(s) \frac{\omega+(\omega+z(\omega+\beta)) s^{\omega+\beta}}{s^{\omega+1}\left(1+s^{\omega+\beta}\right)^{z+1}} d s .
$$

Thus

$$
\liminf _{\lambda \rightarrow 0} \frac{F(\lambda)}{G(\lambda)} \geq(1-\epsilon) \theta^{n}
$$

Letting $\epsilon \rightarrow 0$, one finishes the proof of Lemma 3.1.

Let us go on the proof of Theorem 1.3. It follows from the above Claim and Lemma 3.1 that $F(\lambda) \geq B(\lambda)$ for every $\lambda>0$, that is

$$
\int_{0}^{+\infty}\left(\operatorname{vol}\left[B\left(x_{0}, \theta t\right)\right]-V_{0}(t)\right) \frac{\omega \lambda+(\omega+z(\omega+\beta)) t^{\omega+\beta}}{t^{\omega+1}\left(\lambda+t^{\omega+\beta}\right)^{z+1}} d t \geq 0 .
$$

Letting $\theta \rightarrow 1$, we have

$$
\int_{0}^{+\infty}\left(\operatorname{vol}\left[B\left(x_{0}, t\right)\right]-V_{0}(t)\right) \frac{\omega \lambda+(\omega+z(\omega+\beta)) t^{\omega+\beta}}{t^{\omega+1}\left(\lambda+t^{\omega+\beta}\right)^{z+1}} d t \geq 0, \quad \forall \lambda>0 .
$$

Since $M$ has non-negative Ricci curvature, we have vol $\left[B\left(x_{0}, t\right)\right] \leq V_{0}(t), \forall t>0$. It then follows from (3.11) that $\operatorname{vol}\left[B\left(x_{0}, t\right)\right]=V_{0}(t)$ for almost every $t \geq 0$, and thus every $t \geq 0$ by continuity. Consequently, $M$ is isometric to $R^{n}$ by the equality case in Bishop-Gromov's theorem. This completes the proof of Theorem 1.3.

Proof of Theorem 1.4. Let $p_{t}(x, y)$ be the heat kernel on $M$. By [BCL], there exists a positive constant $C_{0}>0$ such that

$$
\sup _{x, y \in M} p_{t}(x, y) \leq \frac{C_{0}}{t^{n / 2}}, \quad \forall t>0 .
$$

In [LY], Li and Yau showed that there is a positive constant $C_{1}(n)$ depending on the dimension of $M$, such that

$$
C_{1}(n) \leq \liminf _{t \rightarrow \infty} \operatorname{vol}[B(x, \sqrt{t})] p_{t}(x, y), \quad \forall x, y \in M .
$$

Combining the above two inequalities, we know that

$$
\liminf _{r \rightarrow+\infty} \frac{\operatorname{vol}[B(x, r)]}{r^{n}} \geq C_{1} C_{0}^{-1}>0 .
$$

Thus $M$ has large volume growth (cf. [S2]). On the other hand, the work of Druet, Hebey and Vaugon [DHV] implies that the sectional curvature of $M$ is identically zero. A theorem of Marenich and Toponogov [MT] states that a complete Riemannian manifold with non-negative sectional curvature and large volume growth is diffeomorphic 
to a Euclidean space. Combining all these facts, we know that $M$ is isometric to $R^{n}$. This completes the proof of Theorem 1.4.

\section{References}

[AG] U. Abresch and D. Gromoll, On complete manifolds with non-negative Ricci curvature, J. Amer. Math. Soc. 3 (1990), no. 2, 355-374.

[A] M. Anderson, On the topology of complete manifolds of non-negative Ricci curvature, Topology 29 (1990), 41-55.

[B] D. Bakry, L'hypercontractivité et son utilisation en théorie des semigroupes, Lectures on probability theory (Saint-Flour, 1992), 1-114, Lecture Notes in Math. 1581, Springer, Berlin, 1994.

[BCL] D. Bakry, D. Concordet and M. Ledoux, Optimal heat kernel bounds under logarithmic Sobolev inequalities, ESAIM Probab. Statist. 1 (1995/97), 391-407.

[BC] R. L. Bishop, R. J. Crittenden, Geometry of manifolds, Academic Press, New York, 1964.

[CKN] L. A. Caffarelli, R. Kohn and L. Nirenberg, First order interpolation inequalities with weights, Compositio Math. 53 (1984), no. 3, 259-275.

[CL] E. Carlen and M. Loss, Sharp constant in Nash's inequality, Internat. Math. Res. Notices, (1993) no. 7, 213-215

[CX1] M. P. do Carmo and C. Y. Xia, Ricci curvature and the topology of open manifolds, Math. Ann. 316 (2000), no. 2, 391-400.

[CX2] - Complete manifolds with non-negative Ricci curvature and the Caffarelli-KohnNirenberg inequalities, Compositio Math. 140 (2004), no. 3, 818-826.

[CW] F. Catrina and Z. Q. Wang, On the Caffarelli-Kohn-Nirenberg inequalities: sharp constants, existence (and non-existence), and symmetry of extremal functions, Comm. Pure and Applied Math. 54 (2001), no. 2, 229-258.

[Ch] I. Chavel, Riemannian geometry: a modern introduction, Cambrige Univ. Press, 1993.

[CC] J. Cheeger and T. Colding, On the structure of spaces with Ricci curvature bounded below I, J. Diff. Geometry, 46 (1997), no. 3, 406-480.

[CCh] K. S. Chou and C. W. Chu, On the best constants for a weighted Sobolev-Hardy inequality, J. London Math. Soc. 48 (1993), no. 1, 137-151.

[D] E. B. Davies, Heat kernels and spectral theory, Cambridge Univ. Press, 1989.

[DPD1] M. Del Pino and J. Dolbeault, Best constants for Gagliardo-Nirenberg inequalities and applications to nonlinear diffusions, J. Math. Pures Appl. 81 (2002), no. 9, 847-895.

[DPD2] _ , The optimal Euclidean $L^{p}$-Sobolev logarithmic inequality, J. Funct. Anal. 197 (2003), no. 1, 151-161.

[DHV] O. Druet, E. Hebey and M. Vaugon, Optimal Nash's inequalities on Riemannian manifolds: the influence of geometry, Internat. Math. Res. Notices. 14 (1999), no. 14, 735-779.

[G] M. Gromov, Metric structures for Riemannian and non-Riemannian spaces, based on the 1981 French original. With appendices by M. Katz, P. Pansu and S. Semmes. Translated from the French by Sean Michael Bates. Progress in Mathematics, 152. Birkhuser Boston, Inc., Boston, MA, 1999. xx+585 pp.

[HLP] G. H. Hardy, J. .E. Littlewood and G. Pólya, Inequalities, Second edition. Cambridge University Press, 1952.

[H1] E. Hebey, Sobolev spaces on Riemannian manifolds, Lecture Notes in Math. 1635, Springer, 1996.

[H2] Nonlinear analysis on manifolds: Sobolev spaces and inequalities, Courant Lecture Notes in Mathematics, 5, New York University, Courant Institute of Mathematical Sciences, New York, 1999.

[Le] M. Ledoux, On manifolds with non-negative Ricci curvature and Sobolev inequalities, Comm. Anal. and Geom. 7 (1999), no. 2, 347-353.

[Li] P. Li, Large time behavior of the heat equation on complete manifolds with nonnegative Ricci curvature, Ann. of Math. 124 (1986), no. 1, 1-21.

[LY] P. Li and S. T. Yau, On the parabolic kernel of the Schrödinger operator, Acta Math. 156 (1986), no. 3-4, 153-201. 
[L] E. H. Lieb, Sharp constants in the Hardy-Littlewood and related inequalities, Ann. of Math. 118 (1983), no. 2, 349-374.

[MT] V. B. Marenich, V. T. Toponogov, Open manifolds of nonnegative Ricci curvature with rapidly increasing volume, Sibirsk. Mat. Zh. 26 (1985), no. 4, 191-194, 206.

[SY] R. Schoen and S. T. Yau, Lectures on differential geometry, International Press, Cambridge, MA, 1994.

[SS] J. Sha and Z. Shen, Complete manifolds with non-negative Ricci curvature and quadratically non-negatively curved infinity, Amer. J. Math. 119 (1997), no. 6, 1399-1404.

[S1] Z. Shen, On complete manifolds of non-negative kth-Ricci curvature, Trans. Amer. Math. Soc. 338 (1993), no. 1, 289-310.

[S2] Complete manifolds with non-negative Ricci curvature and large volume growth, Invent. Math. 125 (1996), no. 3, 393-404.

[SSO] Z. Shen and C. Sormani, The codimension one homology of a complete manifold with nonnegative Ricci curvature, Amer. J. Math. 123 (2001), no. 3, 515-524.

[SO1] C. Sormani, Non-negative Ricci curvature, linear diameter growth and finite generation of fundamental groups, J. Differential Geom. 54 (2000), no. 3, 547-559.

[SO2] - On loops representing elements of the fundamental group of a complete manifold with nonnegative Ricci curvature, Indiana Univ. Math. J. 50 (2001), no. 4, 1867-1883.

[T] G. Talenti, Best constant in Sobolev inequality, Ann. Mat. Pura Appl. 110 (1976), 353-372.

[W] Q. Wang, A topological rigidity theorem on open manifolds with nonnegative Ricci curvature, Ark. Mat. 42 (2004), no. 2, 399-407.

[X1] C. Xia, Complete manifolds with non-negative Ricci curvature and almost best Sobolev constant, Illinois J. Math. 45 (2001), no. 4, 1253-1259.

[X2] Large volume growth and the topology of open manifolds, Math. Z. 239 (2002), no. $3,515-526$.

[X3] The Gagliardo-Nirenberg inequalities and manifolds of non-negative Ricci curvature, J. Funct. Anal. 224 (2005), no. 1, 230-241.

Departamento de Matemática, Universidade de Brasília, 70910-900, Brasília-DF, Brazil

E-mail address: xia@mat.unb.br 